\title{
Large and Small vessel Severe Involvement in ANCA-Associated Vasculiitis
}

\author{
Javier Villacorta ${ }^{1^{*}}$, Francisco Díaz-Crespo ${ }^{2}$, Ramona Stanescu ${ }^{3}$ and Gema Fernandez-Juarez ${ }^{1}$ \\ ${ }^{1}$ Nephrology Division of Hospital Fundacion Alcorcon, Alcorcon, Spain \\ ${ }^{2}$ Pathology Division Hospital Virgen de la Salud, Toledo, Spain \\ ${ }^{3}$ Pathology Division of Hospital Fundacion Alcorcon, Alcorcon, Spain \\ "Corresponding-author: Javier Villacorta, Nephrology Division Hospital Universitario Fundación Alcorcon, Calle Budapest 1. 28922, Alcorcón. Comunidad de Madrid, \\ Spain, Tel: 34627313534; E-mail: jvillacorta@fhalcorcon.es
}

Received date: April 4, 2016; Accepted date: April 25, 2016; Published date: April 28, 2016

Copyright: @ 2016 Villacorta J, et al. This is an open-access article distributed under the terms of the Creative Commons Attribution License, which permits unrestricted use, distribution, and reproduction in any medium, provided the original author and source are credited.

\section{Case Report}

A 63 years-old man was admitted with two weeks history of weakness, generalized arthralgias and mild dyspnea. He had a previous history of gastric ulcer treated with gastroduodenal bypass. His physical examination at admission revealed blood pressure of 130/80 $\mathrm{mm} \mathrm{HG}$, temperature of $36^{\circ} \mathrm{C}$, some bronchial breath sounds without crackles and trace edemas in both lower extremities. The initial laboratory data showed hemoglobin of $8,7 \mathrm{~g} / \mathrm{L}$, white normal blood cell and platelet count. Acute renal failure was documented with creatinine of $601 \mathrm{mmol} / \mathrm{L}$, without electrolytes disturbances. His urine analysis reveled $1+$ protein and $>100 \mathrm{RBC} / \mathrm{hpf}$ without cellular casts. Antineutrophil cytoplasmic antibodies (ANCAs) test was performed and was positive for cytoplasmic pattern (c-ANCA) with ELISA test showing anti- PR3 activity $(177 \mathrm{mU} / \mathrm{ml})$. He was tested for complement, immunoglobulins, cryglobulins, antinuclear antibodies, antiDNA and anti-glomerular basement membrane antibodies, with normal results. Serologic tests did not exhibit evidence of infection with hepatitis B or C virus. Otorhinolaryngology examination did not show remarkable lesions. Given the presence of pulmonary infiltrates on chest radiography at admission a chest computerized tomography (CT) scan was performed showing bilateral alveolar infiltrates suggestive of diffuse alveolar haemorrhage, which was confirmed with bronchoscopy. Renal biopsy showed pauci-immune focal necrotizing glomerulonephritis with extracapillary cellular proliferation affecting $55 \%$ of glomeruli. Since c- ANCA vasculitis disease with pulmonary and renal involvement was diagnosed, early treatment with corticosteroids, plasmapheresis, and cyclophosphamide was initiated. $\mathrm{He}$ received seven daily sessions of plasmapheresis, while haemodialysis was performed on alternate days. Intravenous cyclophosphamide was administered according to the EUVAS group protocol (dosage of $0,75 \mathrm{gr} / \mathrm{m}^{2}$ of body surface area administered every three weeks), with careful dose adjustment to renal function. After the seventh day of treatment the patient experienced respiratory improvement with stabilization in haemoglobin levels and progressive radiological resolution of pulmonary infiltrates. He also experienced progressive increase in urine output and improvement in renal function, being able to discontinue haemodialysis. Fifteen days after admission the patient presented sudden abdominal pain leading to anaemia and haemorrhagic shock. An urgent CT scanner was performed and evidenced the presence of hemoperitoneum due to spontaneous rupture of a gastroduodenal artery aneurysm. Emergency surgery was performed with gastroduodenal artery ligation. CT scan also showed spindle shape aneurysms at the origin of the celiac trunk (13 $\mathrm{mm}$ diameter), the superior mesenteric artery (16 $\mathrm{mm}$ diameter), splenic artery $(11 \mathrm{~mm})$ and left colic branch $(1 \mathrm{~cm})$, with spindle ectasia of both iliac arteries (Figure 1). Aneurysm histology showed necrotizing vasculitis of the vessel wall with fibrinoid necrosis and transmural neutrophilic inflammatory infiltrate, without granulomas (Figure 2). The patient had a satisfactory postoperative recover without complications, and continued with immune-suppressive therapy. Other vascular lesions screening was performed with PET-scan which showed only inflammatory activity at the known aneurysms. Two months after induction treatment he had experienced was in complete remission of pulmonary capillaritis and glomerulonephritis. At this point, a control CT scan was performed that showed a progression on visceral aneurysmatic lesions; mesenteric artery aneurysm had grown (23 $\mathrm{mm}$ of diameter) and developed a thrombus into the artery wall. Large vessel vasculitis involvement was considered treatment resistant, thus high dose steroids treatment was maintained and a second line immune-suppression therapy with rituximab was proposed. Four weekly doses of rituximab $\left(375 \mathrm{mg} / \mathrm{m}^{2}\right)$ were administered. One year after ANCA vasculitis was diagnosed, the patient remains under remission of the glomerulonephritis, showing normal inflammatory markers, negative serum anti-PR3 antibodies and without evidence of clinical vasculitis activity. A CT was performed every six months for aneurysms evaluation and further enlargement or progression of the lesions has not been observed.

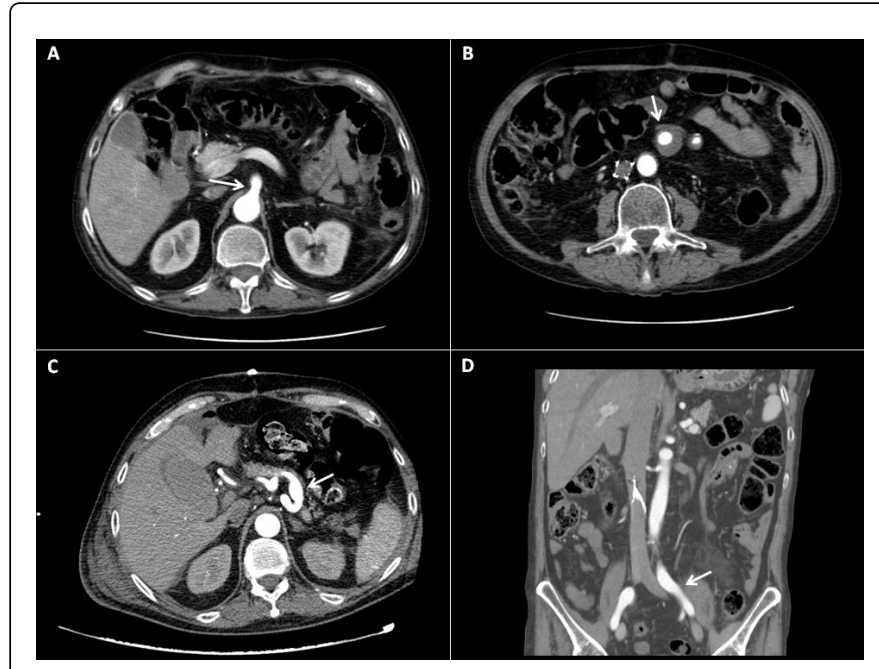

Figure 1: Abdominopelvic computed tomography scan. Panel A: spindle shape aneurysm at the origin of the celiac trunk (arrow). Panel B: aneurysm of superior mesenteric artery (arrow) and colic artery with thrombus narrowing the vessel lumen. Panel C: aneurysm splenic artery (arrow). Panel D: spindle ectasia of both iliac arteries (arrow). 


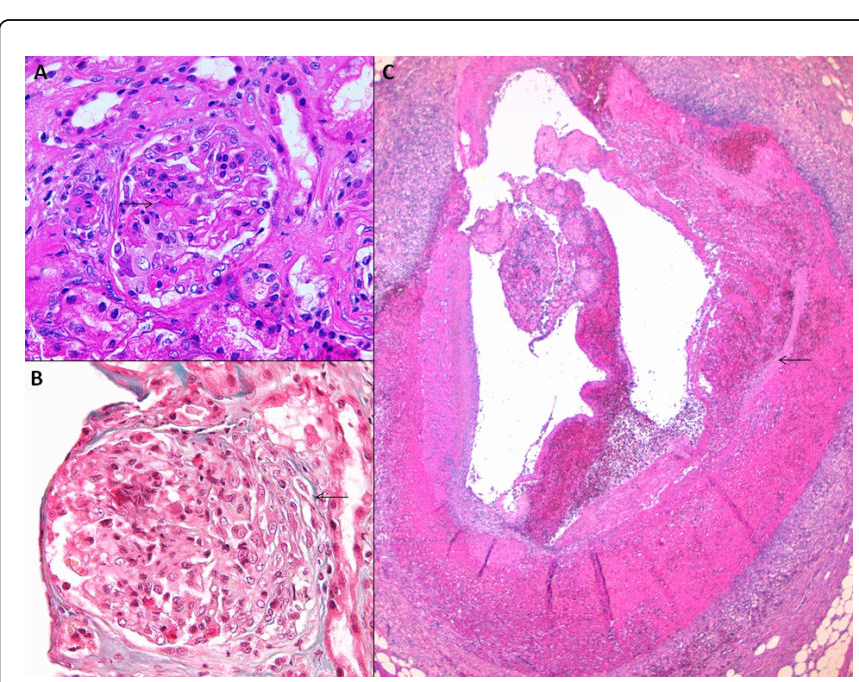

Figure 2: Renal biopsy (A and B) and gastroduodenal aneurysm biopsy (C). Panel A: Hematoxylin-eosin $(\times 40)$. Glomerular cellular proliferation with focal presence of fibrinoid necrosis (arrow). Panel B: Masson's trichromic stain x40). Glomerular extracapillar cellular proliferation (arrow) with small areas with fibrinoid necrosis. Panel C: Hematoxylin-eosin (x10). Gastroduodenal artery. Fibrinoid necrosis of the vessel wall (arrow) plus transmural and periadventitial acute inflammatory infiltrates.

\section{Discussion}

ANCA-associated vasculitis is a group of immune-mediated chronic inflammatory diseases that typically involve small vessels, mainly capillaries, venules and arterioles. The Chapel Hill Consensus Conference (CHCC) classification system for the systemic vasculitis established the distinction between large vessel vasculitis (Giant Cell Arteritis and Takayasu 's disease) defined as predominant involvement of large arteries, mainly the aorta and its main branches; medium vessel vasculitis (Nodose panarteritis (PAN) and Kawasaki disease) defined as predominant involvement of medium size arteries, mainly visceral arteries and their branches; and small vessel vasculitis in which intraparenchymal arteries, arterioles, capillaries and venules are compromised [1]. In small vessel vasculitis the distinction between ANCA associated vasculitis (AAV) versus immune-complex mediated small vessel vasculitis (cryoglobulinemia, IgA vasculitis) was made. The AAV includes granulomatous with polyangitis (GPA), microscopic polyangiitis (MPA) and Churg-Strauss syndrome (CSS) [2]. Renal disease is characterized by the presence of focal necrotizing glomerulonephritis with extracapillary proliferation and inflammation and necrosis of the vessel walls.

Multiple visceral artery aneurysms are typical of c-PAN, however, the presence of glomerular injury and high titres of ANCA in our patient questions this diagnosis [3]. Although slight small-sized vessel involvement could be occasionally observed in patients presenting characteristics of $c$ PAN, we found in this case an equally severe involvement of medium and large size vessels (abdominal visceral branches and iliac arteries), as well as and small vessels (glomerulonephritis and pulmonary capillaritis). On the other hand, some cPAN patients have been reported to show positive tests for ANCAS, last update of CHCC considered the absence of ANCAs as a main characteristic for cPAN diagnosis [4]. In our patient, we observed an intense relationship between PR3-ANCAS serum levels and disease activity, as the title of PR3 was negative after six months of treatment and it decreased in parallel to other inflammatory markers.

The prevalence of large and medium size vessel involvement in ANCA associated vasculitis seems to be low. Guillevin et al. compared the clinical, angiographic, immunological and pathological characteristics of patients with microscopic polyangitis and classic PAN [5]. They found that elevated ANCA titers were only present in $6.5 \%$ of patients with CPAN, and the correlation between abnormal angiograms (medium and large vessel damage) and the presence of ANCAs was very low (less than 10\%). This low incidence of large and medium vessel compromise associated to ANCA vasculitis was also observed in autopsies series of patients with microscopic polyangeitis [6].

Therefore, this case in which small and large vessels are severe compromised does not meet CHCC criteria and vascular disease could be attributed either to a small and large vessel vasculitis overlap syndrome, or as a part of the spectrum of AAVs with an extended vascular compromise. It would be expected that vascular damage could be caused by different immune pathogenic mechanisms, possibly involving antibodies such as ANCAs and anti-endothelial cells antibodies, but also immune cellular dependent mechanisms. The only series reporting large vessel disease in AAV found that these patients had clinical, demographic and histological characteristics that parallel those of the general AAV patients, but differ clinically and pathologically from the classic large vessel vasculitis (Takayasu's disease and giant cell arteritis) [7]. Up to now, treatment proposed for both large and small vessel vasculitis is similar, and it implies immunesuppressive therapy with steroids and cyclophosphamide [8]. Prognostic in our patient is limited due to the presence of multiple and large visceral aneurysms, and such severe extend of vascular lesions in AAV has not been previously reported. We probably cannot expect a regression of the lesions as the elastic fibres of the media are already destroyed resulting in pseudoaneurysm formation, but immunesuppressive therapy can stabilize inflammation process and avoid further enlargement and new lesions. Surgical and endovascular treatment might be necessary in these patients, and it is recommended to be performed when vasculitis activity is controlled with immune suppression therapy in order to obtain better outcome.

An important progress has been made in understanding the pathogenesis of the AAVs. Besides genetic and environmental factors involved in the cause of these diseases, pathogenic pathways, related to both humoral and cellular immune responses, have been elucidated [9]. However, further knowledge of the etiopathogenesis would allow better understanding of these cases that don't fulfil current classifications criteria, and could open the possibility for new classifications based not only on clinical features or extent of the lesions, but also on the immune-pathogenic mechanisms involved. Considering its low incidence, we believe that screening radiological tests for large vessel involvement are not necessary. However, this type of damage is a possible pattern that clinicians should always take into account when evaluating ANCA positive patients.

\section{References}

1. Jennette JC, Falk RJ, Bacon PA, Basu N, Cid MC, et al. (2013) 2012 revised International Chapel Hill Consensus Conference Nomenclature of Vasculitides. Arthritis Rheum 65: 1-11. 
Citation: Villacorta J,Díaz-Crespo F,Stanescu R,Fernandez-Juarez G (2016) Large and Small vessel Severe Involvement in ANCA-Associated Vasculiitis. J Vasc 2: 105. doi:10.4172/2471-9544.100105

Page 3 of 3

2. Jennette JC, Falk RJ, Andrassy K, Bacon PA, Churg J, et al. (1994) Nomenclature of systemic vasculitides. Proposal of an international consensus conference. Arthritis Rheum 37: 187-192.

3. Kirkland GS, Savige J, Wilson D, Heale W, Sinclair RA, et al. (1997) Classical polyarteritis nodosa and microscopic polyarteritis with medium vessel involvement--a comparison of the clinical and laboratory features. Clin Nephrol 47: 176-180.

4. Tanaka M, Matsuo K, Nakamura H, Ishikawa S, Matsuyama K (2006) [Two cases of classical polyarteritis nodosa associated with MPOANCA]. Nihon Jinzo Gakkai Shi 48: 371-376.

5. Guillevin L, Lhote F, Amoroux J (1996) Antineutrophil cytoplasmic antibodies, abnormal angiograms and pathological findings in Polyarteritis Nodosa and Churg-Strauss Syndrome: Indications for the classification of vasculitides of the Polyarteritis Nodosa Group. British Journal of Rheumatology 35: 958-964.

6. Rott T, Vizjak A, Koselj M (2000) ANCA-associated vasculitis--an autopsy study. Wien Klin Wochenschr 112: 671-675.

7. Chirinos JA, Tamariz LJ, Lopes G, Del Carpio F, Zhang X, et al. (2004) Large vessel involvement in ANCA-associated vasculitides: report of a case and review of the literature. Clin Rheumatol 23: 152-159.

8. Hamour S, Salama AD, Pusey CD (2010) Management of ANCAassociated vasculitis: Current trends and future prospects. Ther Clin Risk Manag 6: 253-264.

9. Kallenberg CG, Stegeman CA, Abdulahad WH, Heeringa P (2013) Pathogenesis of ANCA-associated vasculitis: new possibilities for intervention. Am J Kidney Dis 62: 1176-1187. 